\title{
Anti-ganglioside complex IgM antibodies in multifocal motor neuropathy and chronic immune-mediated neuropathies
}

\author{
Eduardo Nobile-Orazio ${ }^{\mathrm{a}, \mathrm{b}, *}$, Claudia Giannotta $^{\mathrm{b}}$, Chiara Briani $^{\mathrm{c}}$ \\ a Department of Translational Medicine, Milan University, Milan, Italy \\ b 2nd Neurology, IRCCS Humanitas Clinical Institute, Rozzano, Milan, Italy \\ c Department of Neurosciences, Padua University, Padua, Italy
}

\section{A R T I C L E I N F O}

\section{Article history:}

Received 14 August 2009

Received in revised form 6 November 2009

Accepted 13 November 2009

\section{Keywords:}

Multifocal motor neuropathy

CIDP

Ganglioside complex

GM1

GD1a

$\operatorname{IgM}$

\begin{abstract}
A B S T R A C T
Anti-ganglioside complexes (GSCs) IgG antibodies have been reported in patients with Guillain-Barré (GBS) or Fisher syndrome but little is known on their presence in multifocal motor neuropathy (MMN) or other chronic immune-mediated neuropathies. We examined 24 patients with MMN, 34 with chronic inflammatory demyelinating polyradiculoneuropathy (CIDP), 23 with neuropathy associated with IgM monoclonal gammopathy (PN + IgM), 13 with GBS, 34 with motor neuron disease (MND), 24 with other neuropathies and 20 normal subjects. Patients' sera were tested by ELISA for IgM reactivity to GM1, GM2, GD1a, GD1b and GT1b and with GSCs made by any combination of two of these gangliosides. In all GM1 positive patients with MMN (11), PN + IgM (1), CIDP (1) and POEMS (1), binding to GM1 was abolished or consistently reduced when tested in GSCs also containing GD1a or other gangliosides. This only occurred in one of the three GM1 positive MND patients. In a patient with PN-IgM and anti-GM2 and GD1a IgM, both reactivities were reduced when tested in GSCs also containing GM1. New reactivities were found in a patient with CIDP and anti-GD1b IgM who presented an additional reactivity to GT1b/GM1 and GT1b/GM2 GSCs, and in one with PN-IgM who had reactivity to GM2/GD1b but not to individual gangliosides. Testing for IgM antibodies to GSCs rarely permitted to identify new reactivities in chronic immune neuropathies. IgM binding to gangliosides was however often modified in GSCs suggesting that these reactivities may be affected by contiguous gangliosides possibly influencing their pathogenicity.
\end{abstract}

(c) 2009 Elsevier B.V. All rights reserved.

\section{Introduction}

Anti-ganglioside GM1 IgM antibodies have been reported in 30-50\% of patients with multifocal motor neuropathy (MMN) and, less frequently, with other immune neuropathies or motor neuron disease (MND) (van Schaik et al., 1995). The pathogenic role of these antibodies in MMN is still unclear as well as what causes the disease in MMN GM1 negative patients. It is also unclear how similar antibodies may be associated with and potentially responsible for different diseases (Nobile-Orazio, 2001).

Recently, several reports described the presence of IgG antibodies against complexes of different gangliosides, called anti-ganglioside complexes (GSCs) antibodies, in patients with Guillain-Barré or Fisher syndrome (Kaida et al., 2004, 2006). In these patients antibodies to GSCs have been detected in approximately $5 \%$ of patients not bearing antibodies to the individual gangliosides forming the complexes (positive interaction). At the same time in some

\footnotetext{
* Corresponding author. FAAN, 2nd Neurology, Milan University, IRCCS Humanitas Clinical Institute, Via Manzoni 56, 20089, Rozzano, Milan, Italy. Tel.: + 39 0282242209; fax: +390282242298 .

E-mail address: eduardo.nobile@unimi.it (E. Nobile-Orazio).
}

patients the mixture of some gangliosides blocked the reactivity to the individual ganglioside forming the GSCs (negative interaction). In these patients reactivity to GSCs was variably related to some clinical features (Kaida et al., 2007, 2008a,b). Little is known on the presence of anti-GSCs IgM antibodies in MMN or other chronic immunemediated neuropathies.

\section{Material and methods}

\subsection{Patients}

We examined 172 patients with MMN or other immune or nonimmune neuropathies and related diseases recruited and tested for anti-nerve antibodies at our Neuropathy Clinics since 2004. All patients had serum collected at the time of other blood tests and they all consented to its use for research. The Internal Review Board of our Institutions approved the study. Twenty four consecutive patients had MMN diagnosed according to the criteria of the European Federation of Neurological Societies and the Peripheral Nerve Society (EFNS/PNS) (Joint Task Force of the EFNS and the PNS, 2006) and 34 had chronic inflammatory demyelinating polyradiculoneuropathy (CIDP) (Joint Task Force of the EFNS and the PNS, 2005). We also tested 23 patients 


\begin{tabular}{|c|c|c|c|c|c|c|}
\hline & GM1 & GM2 & GD1a & GD1b & GT1b & BSA \\
\hline GM1 & $x^{2}$ & & 1 & & 1 & \\
\hline GM2 & & X & ? & & $i$ & \\
\hline GD1a & - & & $X$ & & 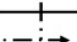 & \\
\hline GD1b & $\ldots$ & 1 & & & - & \\
\hline$\overline{\text { GT1b }}$ &.--1 & $-1 .-$ & $\ldots-$ & $+\cdot \cdot$ & 1. & \\
\hline BSA & & & & & & \\
\hline
\end{tabular}

Fig. 1. Scheme used to test for anti-ganglioside complex (GSCs) IgM antibodies by ELISA. Patients' sera were tested with individual gangliosides $(0.5 \mu \mathrm{g})$ and with the combination of two gangliosides ( $0.5 \mu \mathrm{g}$ each). All the wells in the first line and column were coated with GM1, those in the second line and column with GM2, the third line and column with GD1a, the fourth line and column with GD1b and the fifth line and column with GT1b. Wells in the last line and column were only coated with bovine serum albumin. The wells under the diagonal line only contained the single ganglioside GM1, GM2, GD1a, GD1b or GT1b.

with neuropathy associated with IgM monoclonal gammopathy with antibodies to the myelin-associated glycoprotein (MAG) (>1/3200 by immunoblot) (19 patients), to sulfatide ( $>1 / 16,000$ by ELISA) (two patients) or to sulfatide, GM2 and GD1a (>1/1280 by ELISA) (one patient), 13 with GBS, 34 with motor neuron disease (MND), 24 with neuropathy of other or undetermined causes including one with POEMS and anti-GM1 IgM, and 20 normal subjects (NS).

\subsection{Methods}

Patients' sera were tested by Enzyme Linked Immunosorbent Assay (ELISA) for IgM reactivity to the individual gangliosides GM1, GM2, GD1a, GD1b and GT1b (0.5 $\mu$ g/well for of each ganglioside) as previously reported (Nobile-Orazio et al., 2008). To better correlate the results obtained with ganglioside complexes, we slightly modified the procedure by testing patients' sera at the dilution of 1:200 and by considering positive the sera when the difference in the absorbance between wells coated with the ganglioside and bovine serum albumin (BSA) exceed 0.100 . All sera were also tested for IgM reactivity to GSCs by coating individual wells with combinations of two gangliosides at the concentration of $0.5 \mu \mathrm{g}$ of ganglioside per well. The scheme of the ELISA plate used to test for anti-GSCs antibodies is illustrated in Fig. 1. We arbitrarily considered that IgM reactivity to gangliosides was positively affected by GSCs if the absorbance in GSCs coated wells exceeded by at least $100 \%$ with a minimum absorbance of 0.200 , the sum of the absorbance of wells coated with the individual ganglioside forming GSCs (positive interaction), and that it was negatively affected if the absorbance from GSCs coated wells was reduced by at least 50\% compared to the absorbance of wells coated with the ganglioside forming the GSCs (negative interaction). All sera were tested in duplicate and the results were always confirmed in a second separate experiment.

To determine whether the reduced IgM binding to GM1 in GSCs containing GM1 could be caused by a reduced binding of GM1 to ELISA wells determined by the other gangliosides forming the GSCs, we tested IgM binding to GSCs prepared by adding to GM1 increasing amount of GD1a (from 0.25 to $1 \mu \mathrm{g}$ per well).

\section{Results}

Eleven patients with MMN (46\%) had serum IgM reactivity to GM1 (8) or to GM1 and GM2 (3). IgM reactivity to GM1 was also found in one patient with CIDP (3\%), one with PN-IgM and anti-MAG reactivity (5\%) and three with MND (9\%). One patient with CIDP had a selective IgM reactivity to GD1b, and one with PN-IgM to GM2 and GD1a.

In all eleven positive patients with MMN, IgM reactivity to GM1 was removed or substantially reduced (negatively affected) when tested in the GSC GM1-GD1a (Fig. 2): in nine of them this reduction exceed $80 \%$ and in two $50 \%$. In ten of them reactivity was also reduced in GM1-GD1b or GM1-GT1b or both and in four also in GM1-GM2. A similar negative effect was observed in the GM1 positive patients with PN + IgM, CIDP and POEMS, with anti-GM1 reactivity reduced in GM1-GD1a, GM1-GD1b and in the first two patients also in GM1-GM2 and GM1-GT1b GSCs. This effect was most evident in patients with high anti-GM1 reactivity, but was also observed and reproducible in patients with low $(<0.200)$ anti-GM1 reactivity (Fig. 2). In one of the three GM1 positive patients with MND, IgM reactivity to GM1 was not affected when tested in any of the GSCs containing GM1 (no interaction) (Fig. 2, MND1), while it was almost abolished in one (MND2), and reduced by almost $50 \%$ in another (MND3). In the patient with PN-IgM and anti-GM2 and -GD1a IgM (and GT1b), both reactivities were markedly reduced when GM2 was complexed with GM1, and GD1a with GM1 or GD1b (Fig. 3a). A clearly positive interaction determined by GSCs was only observed in two patients. One was a 67-year-old man with a 9-year history of demyelinating sensory neuropathy with a few superimposed post-infective severe motor relapses that always improved after high-dove intravenous immunoglobulin therapy. This patient had a selective IgM reactivity to GD1b that was not affected by other gangliosides, while IgM reactivity to GM1 and GM2 only appeared in GSCs also containing GT1b (positive interaction) (Fig. 3b). The other patient was a 65-year-old man with 23-year history of predominantly motor neuropathy, IgM kappa biclonal gammopathy and marginally increased anti-MAG IgM antibodies. This patient had a

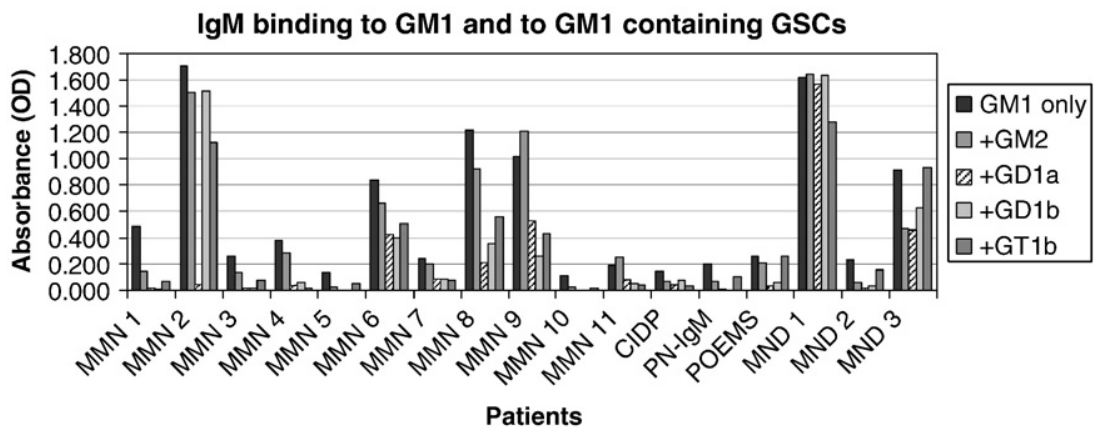

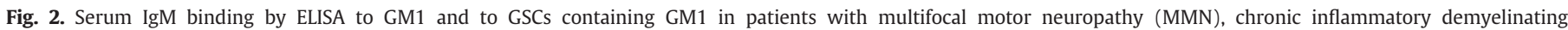

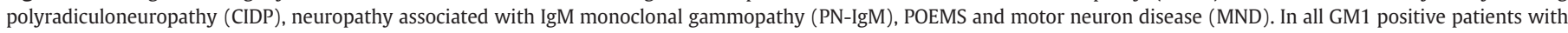

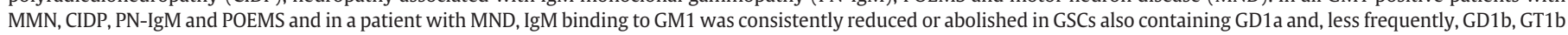
and GM2. IgM binding to GM1 was not reduced in a patient with MND and was partially reduced in another one. 

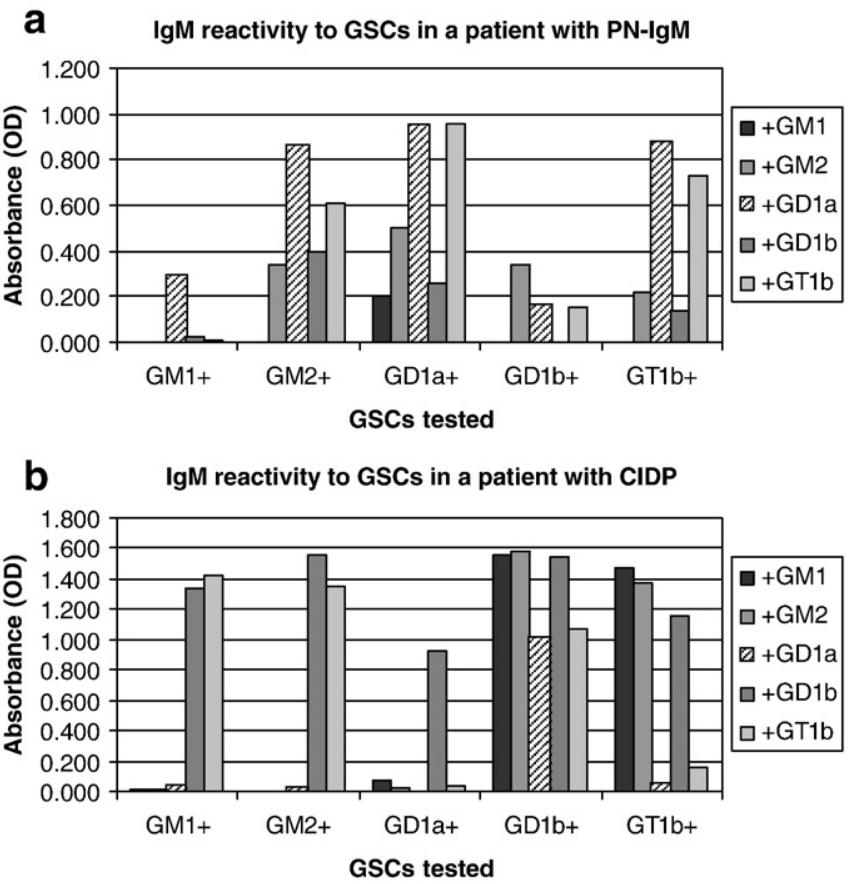

Fig. 3. Serum IgM binding by ELISA to gangliosides and to GSCs in one patient with PN-IgM (a) and one with chronic inflammatory demyelinating polyradiculoneuropathy (CIDP) (b). In PN-IgM, anti-GM2 and -GD1a IgM reactivities were markedly reduced when GM2 was complexed with GM1, and GD1a with GM1 or GD1b. In CIDP, IgM intensely react with GD1b and this reactivity was not or was only marginally affected in any GSCs containing GD1b; an intense IgM reactivity also appeared with the GSCs GM1/GT1b and GM2/GT1b in the absence of IgM reactivity to the individual gangliosides GM1, GM2 and GT1b.

consistent IgM reactivity to GM2/GD1b that was specific for the kappa light chain, but not to the individual gangliosides.

To determine whether reduced IgM binding to GM1 could be caused by a reduced binding of GM1 to ELISA plates caused by the other gangliosides forming the GSCs, we tested IgM binding to GM1 in the presence of increasing doses of GD1a ( 0.25 to $1 \mu \mathrm{g})$. In MMN and PN-IgM patients, binding to GM1 was markedly reduced by minimal amount of GD1a while the same amount of GD1a only marginally affected GM1 binding in the two MND patients tested (Fig. 4).

\section{Discussion}

We examined patients with MMN and other chronic immunemediated neuropathies to determine whether testing for antibodies to GSCs may reveal the presence of new antibody reactivities undetect-

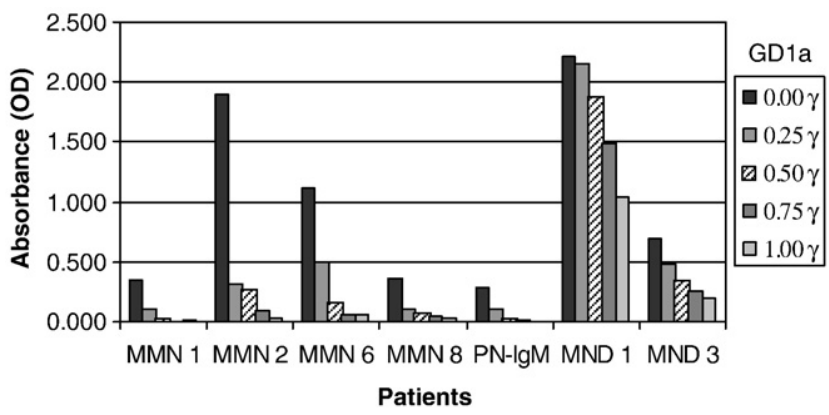

Fig. 4. Effect on IgM reactivity to GM1 of increasing amount of GD1a in the GSC GM1/GD1a In patients with MMN and PN-IgM IgM reactivity to GM1 was reduced by small amounts of GD1a, while a partial reduction of reactivity only appeared with much higher concentration of GD1a in patients with MND $(\gamma=\mu \mathrm{g})$. able with the current ELISA procedure for antibodies to individual gangliosides. In only one patient with CIDP and high titers of antibodies to GD1b and one with PN-IgM we found that testing for IgM antibodies to GSCs allowed to detect an additional intense IgM reactivity to the complexes formed by GM1 or GM2 with GT1b, as previously reported in a few patients with GBS and high anti-GD1b IgG (Kaida et al., 2008a), or by GM2 and GD1b, respectively, in the absence of reactivity to the individual gangliosides. No other patient showed new reactivities by this procedure indicating that, at odds with GBS and FS where approximately $5-10 \%$ of the patients were found to have antibody to GSCs in the absence of antibodies to individual gangliosides (Kaida et al., 2006, 2007, 2008b), this seldom occurred for IgM antibodies in patients with MMN or other chronic immune neuropathies.

Testing for anti-GSCs IgM antibodies in our patients with increased anti-GM1 antibodies let us identify different patterns of reactivities. In all 11 GM1 positive patients with MMN, and in those with PN-MAG, CIDP and POEMS, IgM reactivity to GM1 was consistently reduced when tested in GSCs also containing GD1a and, less frequently or less intensely, GD1b, GM2 and GT1b. This only occurred in one of the three GM1 positive patients with MND while in the other two patients, antiGM1 IgM reactivity was moderately or not affected. This "negative" effect on IgM binding to GM1 in MMN was not dependent on the blocking of GM1 binding to the ELISA wells determined by the other gangliosides in GSCs as this only occurred in MMN, but not in all MND, with minimal amount of GD1a. A similarly "negative" effect on IgM binding to GM2 and GD1b in GSCs also containing GM1 was observed in a patient with PN-IgM. These "negative" effects on antibody binding to gangliosides have been previously reported in GBS (Kaida et al., 2008a), suggesting that the interaction of different gangliosides may lead not only to the formation of new antigens but also to the hiding of reactive antigens (Willison, 2005). If we suppose indeed that variable proportions and contiguities of individual gangliosides may be expressed in different neural membrane, it is tempting to speculate that this may lead to the formation of new antigens but also to the different expressions or hiding of reactive epitopes in different neural membranes. A brilliant support to this hypothesis was recently given by Greeshields et al. (2009) who showed that the GM1 binding epitope may be often hidden in mice membranes by contiguous gangliosides and particularly by GD1a, so that the pathogenic effect of anti-GM1 antibodies may vary depending on recognition of exposed or cryptic antigens. This may theoretically help in explaining the different pathogenic effects of apparently similarly reacting antiganglioside antibodies. In this study we found indeed a different pattern of reactivity between GM1 positive sera from MMN and some MND patients. Whether, however, these antigenic modifications also occur on human neural membranes is not known but, if this was the case, it would offer a clue to explain the different pathogenic effect in vivo of similarly in vitro reacting IgM antibodies.

\section{References}

Greeshields, K.N., Halstead, S.K., Zitma, F.M.P., Rinaldi, S., Brennam, K.M., O'Leary, C., Chamberlain, L.H., Easton, A., Roxburgh, J., Pediani, J., Furukawa, K., Furukawa, K., Goodyear, C.S., Plomp, J.J., Willison, H.J., 2009. The neuropathic potential of antiGM1 autoantibodies is regulated by the local glycolipids environment in mice. J. Clin. Invest. 119, 595-609.

Joint Task Force of the EFNS and the PNS, 2005. European Federation of Neurological Societies/Peripheral Nerve Society Guideline on management of chronic inflammatory demyelinating polyradiculoneuropathy. J. Periph. Nerv. Sys. 10, 220-228.

Joint Task Force of the EFNS and the PNS, 2006. European Federation of Neurological Societies/Peripheral Nerve Society Guideline on management of multifocal motor neuropathy. J. Periph. Nerv. Sys. 11, 1-8.

Kaida, K., Morita, D., Kanzaki, M., Kamakura, K., Motoyoshi, K., Hirakawa, M., Kusunoki, S., 2004. Ganglioside complexes as new target antigens in Guillain-Barré syndrome. Ann. Neurol. 56, 567-571.

Kaida, K., Kanzaki, M., Morita, D., Kamakura, K., Motoyoshi, K., Hirakawa, M., Kusunoki, S., 2006. Anti-ganglioside complex antibodies in Miller Fisher syndrome. J. Neurol. Neurosurg. Psychiatry 77, 1043-1046. 
Kaida, K., Morita, D., Kanzaki, M., Kamakura, K., Motoyoshi, K., Hirakawa, M., Kusunoki, S., 2007. Anti-ganglioside complex antibodies associated with severe disability in GBS. J. Neuroimmunol. 182, 212-218.

Kaida, K., Kamakura, K., Ogawa, G., Ueda, M., Motoyoshi, K., Arita, M., Kusunoki, S. 2008a. GD1b specific antibody induces ataxia in Guillain-Barré syndrome. Neurology 71, 196-201.

Kaida, K., Sonoom, M., Ogawa, G., Kamakura, K., Ueda-Sada, M., Arita, M., Motoyoshi, K., Kusunoki, S., 2008b. GM1/GalNAc-GD1a complex. A target for pure motor GuillainBarré syndrome. Neurology 71, 1683-1690.
Nobile-Orazio, E., 2001. Multifocal motor neuropathy. J. Neuroimmunol. 115, 4-18.

Nobile-Orazio, E., Gallia, F., Terenghi, F., Allaria, S., Giannotta, C., Carpo, M., 2008. How useful are anti-neural IgM antibodies in the diagnosis of chronic immune-mediated neuropathies? J. Neurol. Sci. 266, 156-163.

van Schaik, I.N., Bossuyt, P.M.M., Brand, A., Vermeulen, M., 1995. Diagnostic value of GM1 antibodies in motor neuron disorders and neuropathies: a meta-analysis. Neurology 45, 1570-1577.

Willison, H., 2005. Ganglioside complexes: new autoantibody targets in Guillain-Barré syndromes. Nat. Clin. Pract. Neurol. 1, 2-3. 